\title{
Superior Mesenteric Vein-First Approach for Robotic Salvage Surgery with Indocyanine Green Fluorescence Angiography
}

\author{
Yun Yang, MD, PhD ${ }^{1}$, Oliver Peacock, BMBS, PhD $^{1}$, Songphol Malakorn, MD ${ }^{1}$, Thitithep Limvorapitak, MD ${ }^{1}$, \\ Syed Nabeel Zafar, MD, MPH ${ }^{1}$, and George J. Chang, MD, MS ${ }^{1,2}$ (i) \\ ${ }^{1}$ Department of Surgical Oncology, University of Texas MD Anderson Cancer Center, Houston, TX; ${ }^{2}$ Department of \\ Health Services Research, University of Texas MD Anderson Cancer Center, Houston, TX
}

\begin{abstract}
Introduction. Inadequate lymphadenectomy is still a major concern in colon cancer surgery. The superior mesenteric vein (SMV)-first approach is a novel, standardized, reproducible method for robotic complete mesocolic excision surgery.

Objective. Our aim was to present the application of the SMV-first approach principles to facilitate robotic salvage surgery for recurrent disease within the mesocolon.

Methods. A 78-year-old female presented with a malignant lymph node deposit within residual right mesocolonic tissue, approximately 3 months following a laparoscopic right hemicolectomy for colon cancer. Dissection was initiated with a transverse curvilinear incision along the inferior aspect of the remaining ileocolic pedicle to identify the SMV. Dissection continued along the ventral aspect of the SMV in a cephalad direction to identify and expose the middle colic vessels at their origin. The use of idocyanine green (ICG) confirmed the vascular anatomy, demonstrating the right branch of the middle colic artery traversing the malignant deposit in the residual mesocolon. Following ligation at the origin of the right branch of the middle colic and ileocolic vessels, the retro-mesocolic plane dissection was completed to excise the malignant deposit and the residual mesocolon.
\end{abstract}

Electronic supplementary material The online version of this article (https://doi.org/10.1245/s10434-020-08222-1) contains supplementary material, which is available to authorized users.

(C) Society of Surgical Oncology 2020

First Received: 9 October 2019;

Published Online: 6 March 2020

G. J. Chang, MD, MS

e-mail: gchang@mdanderson.org
Results. The patient was discharged home the following day. The pathological specimen confirmed metastatic poorly differentiated adenocarcinoma in one of nine lymph nodes, and the vascular pedicle resection margin was negative for tumor.

Conclusion. Following the SMV-first approach principles provides a safe plane for dissection, and, in conjunction with ICG, facilitates the delineation of the vascular anatomy, to enable robotic salvage surgery to be performed. ${ }^{1-3}$

FUNDING No sources of support.

DISCLOSURE Dr. George J. Chang is a consultant for MOREHealth and Medicaroid Corporation. Yun Yang, Oliver Peacock, Songphol Malakorn, Thitithep Limvorapitak, and Syed Nabeel Zafar have no conflicts of interest to declare.

\section{REFERENCES}

1. Bertelsen CA, Neuenschwander AU, Jansen JE, et al. Disease-free survival after complete mesocolic excision compared with conventional colon cancer surgery: a retrospective, population-based study. Lancet Oncol. 2015;16:161-168.

2. Sammour T, Malakorn S, Thampy R, et al. Selective central vascular ligation (D3 lymphadenectomy) in patients undergoing minimally invasive complete mesocolic excision for colon cancer: optimizing the risk-benefit equation. Colorectal Dis. 2020;22(1):53-61.

3. Yang Y, Malakorn S, Zafar SN, et al. Superior mesenteric veinfirst approach to robotic complete mesocolic excision for right colectomy: technique and preliminary outcomes. Dis Colon Rectum. 2019;62:894-897.

Publisher's Note Springer Nature remains neutral with regard to jurisdictional claims in published maps and institutional affiliations. 